\title{
La cholestyramine, médicament du prurit des ictériques
}

Les démangeaisons imposent aux ictériques chroniques par retention un supplice atroce, bien pire que toutes les douleurs, empêchant complètement le repos et conduisant parfois au suicide les malchan-ceux que la médecine était jusqu'ici impuissante à soulager.

On recommandait autrefois les méthodes dites de désensibilisation, l'hydro- ou la balnéothérapie, les anti-histaminiques de synthèse, la colchicine. Inversement, Гiode, Tantipyrine, les bromures, les barbi-turiques étaient déconseillés.

Plus récemment, on a préconisé les cortícostéroïdes, très discutés car ils n'améliorent pas la fonction d'excrétion biliaire, ni la pente P2 d'élimination de la B.S.P.; les hormones sexuelles, en particulier la méthyltestostérone et le noréthandrolone (Nilevar), qui favorisent malheureusement l'élévation des taux de bilirubine et d'acides biliai-res dans le serum (effet cholestatique); enfin les antíbíotíques agissent utilement sur Гinfection (cholangite) surajoutée, et certains d'entre eux (Néomycine, par exemple) seraient capables de quadrupler $\Gamma$ ex-crétion fécale des acides biliaires non conjugués.

Néanmoins, certaines observations cliniques répétées, comme par exemple Гeffet étonnant des drainages, ont fait admettre que la réabsorption de la bile était un facteur pathogénique du prurit plus important que le taux de la cholalémie, incriminé d'habitude.

D'où $\Gamma$ idée de chercher à bloquer le circuit entero-hêpatíque par une résine insoluble, échangeuse d'ions et capable de lier les acides biliaires, in vivo comme in vitro, empêchant leur réabsorption et favorisant leur elimination fécale. Outre ce mécanisme, le fait de dériver la bile à Textérieur multiplie par 15 la cholérèse (stimulation de la cholérèse par Tabsence d'acides biliaires dans le système porte), selon S. Berg-ström (Fed. Proc. 20: 28 [1962]); les chélateurs des acides biliaires agiront done comme si l'on créait une «fistule biliaire médicale».

C'est en 1960 que les premiers travaux concernant la cholestyramine (forme chlorée d'une résine échangeuse d'anions, fortement basique) ont été publiés par J. B. Carey, jr. (J. lab. din. Med. 56: 797 [1960]) et par le groupe de Van Itallie (New Engl. J. Med. 265: 469 [1961]). Cette résine est capable de multiplier par 2,6 Гélimination fécale de la bile, et ferait ainsi baisser le taux de la cholalémie.

378

\section{Editorial}

II s'agit d'une substance dénuée de toute toxicité (10 g/ $\mathrm{kg}$ sont in-offensifs pour la souris); et pour qu'on aperçoive, en trois mois de traitement, un désordre électrolytique notable - acidose hypochloré-mique, notamment - il faut en donner au chien de 20 à $40 \mathrm{~g}$. par jour; $10 \mathrm{~g}$ par jour pendant 6 mois sont parfaitement tolérés. Cependant, au cours de traitements prolongés, il faut tenir compte de la possibilité de gene dans $\Gamma$ absorption des vitamines liposolubles (R. E. Vísíntine: Lancet2: 341 [1961]) setraduisant par une tendance aux saignements par hypoprothrombinémie, à prévenir par la prescription de vita-mine $\mathrm{K}$.

Indépendamment de ces perturbations humorales, la cholestyra-mine n'est pas toujours bien supportée: des diarrhées (voire des stéatorrhées) et des nausées ont été rapportées dans presque un quart des cas. Les tablettes chocolatées à $750 \mathrm{mg}$ utilisées par D. V. Datta et Sh. Sherlock 
(Brit. med. J. 1: 216 [1963]) sont mieux acceptées sans doute que la poudre actuelle que nous avons été obliges de faire encap-suler (capsules de $1 \mathrm{~g}$ ).

La cholestyramine a été réservée pendant plusieurs années à la recherche, mais elle est maintenant commercialisée par Merck, Sharp et Dohne sous le nom de Cuemíd, qui contient 82 $\%$ de substance active. Une cuillerée à soupe pleine correspond à $4 \mathrm{~g}$ de poudre. Cette quantité est la dose initiale, à prendre 3 fois par jour, aux repas; il faut la mélanger à un jus de fruit ou sauce de legumes et $\Gamma$ avaler sans tarder, car le melange devient rapidement visqueux. On évitera égale-ment de prendre d'autres medicaments dans les heures qui precedent, la résine pouvant les adsorber et empêcher ainsi leur absorption intestinale. La dose initiale de $3 \times 4 \mathrm{~g}=12 \mathrm{~g}$ de Cuemid ou $10 \mathrm{~g}$ de cholestyramine peut être augmentée, en cas de 11011 soulagement dans les 8 à 10 jours; quand le prurit tend à s'effacer, on réduit au contraire la quantité totale, mais toujours répartie en 3 doses quotidiennes.

D'après son mécanisme d'action, la cholestyramine sera efficace dans les íctères par obstruction partielle et surtout dans celui de la cirrhose bílíaíre primitive. Puisqu'il s'agit d'absorber de la bile au niveau de Tintestin, on cherche à obtenir la cholérèse maximale, par exemple en faisant précéder le repas par du sorbitol.

La medication peut être employee très longtemps: F. Schaffner et coll. (Gastroenterology 48: 293 [1965]) l'ont poursuivie jusqu'à 18 à 42 mois chez 4 malades atteints de cirrhose biliaire primitive. Mais si le prurit est supprimé ainsi de façon durable, transformant l'exis-tence de ces malheureux suppliciés, la cholestyramine n'a guère d'ac-

Editorial

379

tion sur les tests hépatiques, et ne modifie pas Гaspect histologique du foie; et quand la cholestérolémie s'abaisse, c'est le plus souvent momentané. J. Plessíer et Rautureau, M. (Rev. int. Hépat. 15: 229 [1965]) qui en ont publié récemment 16 observations, confirment $\Gamma$ excellente et rapide action sur le prurit (sauf s'il est trop ancien); le taux des acides biliaires baisse de façon «plus spectaculaire» que celui de la bilirubine, mais sans parallèlisme constant avec la disparition du prurit.

Les remarquables résultats obtenus par la cholestyramine parlent en faveur de la responsabilité de la retention des sels biliaires dans la production du prurit des ictériques. Mais on peut imaginer d'autres mécanismes que l'empêchement de la réabsorption intestinale: par exemple une modification dans le métabolisme des acides biliaires; ou encore une action sur une substance de nature stéroïdienne, capable de provoquer le prurit et qui serait éliminée avec la cholestyramine.

Cela expliquerait comment certains ictères par obstruction totale sont parfois, contrairement à ia théorie, améliorés également par l'administration de Cuemid. M.D. 